\title{
6 HEALTHIER CMR PHENOTYPES ARE LINKED TO FAVOURABLE BRAIN MRI STRUCTURE AND FUNCTION METRICS IN THE UK BIOBANK
}

\begin{abstract}
${ }^{1}$ Celeste McCracken*, ${ }^{2}$ Zahra Raisi-Estabragh*, ${ }^{1}$ Betty Raman, ${ }^{4}$ Andrea Dennis, ${ }^{5}$ Thomas E Nichols, ${ }^{6}$ Masud Husain, ${ }^{2,3}$ Steffen E Petersen, ${ }^{1}$ Stefan Neubauer. ${ }^{1}$ Division of Cardiovascular Medicine, Raddiffe Department of Medicine, University of Oxford, National Institute for Health Research Oxford Biomedical Research Centre, Oxford University Hospitals NHS Foundation Trust, Oxford, OX3 9DU, UK; ${ }^{2}$ William Harvey Research Institute, NIHR Barts Biomedical Research Centre, Queen Mary University of London, Charterhouse Square, London, EC1M 6BQ, UK; Barts Heart Centre, St Bartholomew's Hospital, Barts Health NHS Trust, West Smithfield, London, ECIA 7BE, UK; ${ }^{3}$ Health Data Research UK, London, UK; Alan Turing Institute, London, UK; ${ }^{4}$ Perspectum Diagnostics Ltd; ${ }^{5}$ Wellcome Centre for Integrative Neuroimaging (WIN FMRIB), University of Oxford, Oxford, UK, Big Data Institute, University of Oxford, Oxford UK; ${ }^{6}$ Wellcome Centre for Integrative Neuroimaging, University of Oxford, Oxford, UK; Department of Experimental Psychology, University of Oxford, Oxford, UK; Nuffield Department of Clinical Neuroscience, University of Oxford, Oxford, UK; Division of Clinical Neurology, John Radcliffe Hospital, Oxford University Hospitals Trust, Oxford, UK; *These authors have contributed equally and share first authorship
\end{abstract}

\subsection{6/heartjnl-2021-BSCMR.6}

Background Existing work, using indirect health measures, suggests independent links between brain and heart health within disease cohorts. We studied the links between MRI-derived heart and brain structure and function in the UK Biobank population-based cohort.

Methods Image acquisition and analysis was according to predefined protocols. We included the following cardiovascular metrics: left ventricular (LV) stroke volume (LVSV), LV global functional index (LVGFI), LV mass to LV end-diastolic volume ratio (LVM: LVEDV), global longitudinal strain (GLS), left atrial ejection fraction (LAEF), aortic distensibility (AoD). T1 and T2 structural images were used to determine overall brain volume, volume of white matter hyperintensities, and volumes of several subcortical structures. We considered microscopic features of white matter integrity assessed using diffusion tensor imaging (DTI) and neurite orientation dispersion and density imaging (NODDI), across a range of white matter fibre tracts. Associations between heart and brain measures were computed using standardised betas from multivariable linear regression models, adjusted for age, sex, deprivation, obesity, education level, alcohol consumption, diabetes, hypertension, high cholesterol, smoking, physical activity, systolic blood pressure, total cholesterol, and glycosylated haemoglobin.

Results We studied 30,373 participants; average age was 63 \pm 7.5 years-old; $52 \%$ were women. Adverse cardiovascular phenotypes across all metrics were associated with larger volume of white matter hyperintensities (e.g., LV GFI: Beta $=-0.049 ; \quad 95 \% \quad \mathrm{CI}=[-0.060, \quad-0.039] ; \quad \mathrm{p}$-value $=$ $\left.3.82 \times 10^{-20}\right)$. Higher LVSV $($ Beta $=0.031 ; 95 \% \mathrm{CI}=$ $[0.022,0.039] ; \quad \mathrm{p}$-value $\left.=1.57 \times 10^{-12}\right)$ and greater AoD $($ Beta $=0.027 ; 95 \% \quad \mathrm{CI}=\quad[0.016,0.039] ; \quad \mathrm{p}$-value $=$ $5.76 \times 10^{-6}$ ) were associated with larger total grey matter volumes. Higher LV GFI, higher LAEF, and higher AoD were associated with healthier white matter microstructure as per higher neurite density and stronger myelin 
membranes (e.g., fractional anisotropy with LV GFI: Beta= $0.052 ; 95 \% \mathrm{CI}=[0.040,0.064] ;$ p-value $\left.=1.79 \times 10^{-17}\right)$. Healthy white matter microstructure was associated with less concentric LV hypertrophy (LVM: LVEDV: Beta= $-0.051 ; 95 \% \quad \mathrm{CI}=[-0.064, \quad-0.038] ; \quad \mathrm{p}$-value $=$ $\left.2.94 \times 10^{-15}\right)$ and better GLS $($ Beta $=-0.032 ; 95 \% \mathrm{CI}=$ $[-0.044,-0.019] ;$ p-value $\left.=4.89 \times 10^{-7}\right)$.

Conclusion In this large population cohort, we demonstrate independent links between heart and brain health using detailed MRI. Participants with healthier hearts had significantly less brain atrophy, less brain microvascular injury, and healthier neuronal structure.

\section{PREDICTORS OF MYOCARDIAL FIBROSIS AND RESPONSE TO ANTI-FIBROTIC THERAPY IN HEART FAILURE WITH PRESERVED EJECTION FRACTION}

\begin{abstract}
1,2 Gavin A Lewis, ${ }^{3}$ Anna Rosala-Hallas, ${ }^{4}$ Susanna Dodd, ${ }^{5-7}$ Erik B Schelbert, ${ }^{2}$ Simon G Williams, ${ }^{2}$ Colin Cunnington, ${ }^{8}$ Theresa McDonagh, ${ }^{1,2,9}$ Christopher A Miller. ${ }^{1}$ Division of Cardiovascular Sciences, School of Medical Sciences, Faculty of Biology, Medicine and Health, Manchester Academic Health Science Centre, University of Manchester, Oxford Road, Manchester, M13 9PL; ${ }^{2}$ Manchester University NHS Foundation Trust, Southmoor Road, Wythenshawe, Manchester, M23 9LT; ${ }^{3}$ Liverpool Clinical Trials Centre, Clinical Directorate, Faculty of Health and Life Sciences, University of Liverpool (a member of Liverpool Health Partners), Alder Hey Children's NHS Foundation Trust, Liverpool, L12 2AP; ${ }^{4}$ Department of Health Data Sciences, Institute of Population Health, Faculty of Health and Life Sciences, University of Liverpool (a member of Liverpool Health Partners), Block F, Waterhouse Bld, 1-5 Brownlow Street, Liverpool, L69 3GL; ${ }^{5}$ Department of Medicine, University of Pittsburgh School of Medicine, Pittsburgh, PA, USA; ${ }^{6}$ UPMC Cardiovascular Magnetic Resonance Center, Heart and Vascular Institute, Pittsburgh, PA, USA; ${ }^{7}$ Clinical and Translational Science Institute, University of Pittsburgh, Pittsburgh, PA, USA; ${ }^{8}$ King's College Hospital, Denmark Hill, London, SE5 9RS; ${ }^{9}$ Wellcome Centre for Cell-Matrix Research, Division of Cell-Matrix Biology and Regenerative Medicine, School of Biology, Faculty of Biology, Medicine and Health, Manchester Academic Health Science Centre, University of Manchester, Oxford Road, Manchester, M13 9PT
\end{abstract}

\subsection{6/heartjnl-2021-BSCMR.7}

Objectives To identify baseline characteristics that associate with baseline myocardial fibrotic burden, predict change in myocardial fibrosis over a year, and predict response to the novel anti-fibrotic agent, pirfenidone, in patients with heart failure with preserved ejection fraction (HFpEF).

Background Myocardial fibrosis, measured using cardiovascular magnetic resonance extracellular volume (ECV), is associated with adverse outcome in HFpEF. In the PIROUETTE (The Pirfenidone in Patients with Heart Failure and Preserved Left Ventricular Ejection Fraction) trial, pirfenidone reduced myocardial fibrosis.

Methods Amongst patients enrolled in the PIROUETTE trial $(n=107)$, linear regression models were used to assess the relationship between baseline variables and baseline myocardial $\mathrm{ECV}$, and with change in myocardial ECV (week 52 value minus baseline value), adjusting for treatment allocation. Linear regression models were used to identify baseline variables that modified pirfenidone treatment effect.

Results Body mass index, left atrial reservoir strain, haemoglobin and aortic distensibility were associated with baseline ECV in stepwise modelling (table 1). QRS duration, left ventricular mass and presence of an infarct at baseline were associated with an increase in ECV from baseline to week 52 in stepwise modelling. Whilst QRS duration, presence of an infarct, global longitudinal strain and left atrial strain modified the treatment effect of pirfenidone when considered individually, no baseline variable modified treatment effect on multivariable modelling. Conclusions Baseline characteristics were identified that associate with myocardial fibrosis and can predict change in myocardial fibrosis over 1 year. No variables that independently modify the treatment effect of pirfenidone were identified (PIROUETTE, NCT02932566).

\section{INTELLIGENT LOCALISERS: AN INTEGRATED TIME- SAVING DEEP LEARNING SOLUTION FOR THE PLANNING OF CINE IMAGING AND IDENTIFICATION OF UNEXPECTED FINDINGS FROM A SINGLE TRANSAXIAL STACK}

James Howard, Sameer Zaman, Darrel Francis, Graham Cole. National Heart and Lung Institute, Imperial College London

\subsection{6/heartjnl-2021-BSCMR.8}

Background CMR scans typically begin with a transaxial stack for anatomical evaluation, but current strategies require (1) additional localiser images for cine planning and (2) expert staff present to identify unexpected findings and adjust protocols for maximum yield.

We present an integrated deep learning solution to (1) automate scan plane prescription without need for localisers and (2) identify important protocol-changing findings at the earliest stages of the scan.

Methods Task 1: We trained a neural network on 1,300 CMR transaxial stacks to identify the spatial co-ordinates of the cardiac apex and the 6 locations on the mitral annulus corresponding to 2-, 3-, and 4-chamber views. By identifying these points, the network proposed long-axis scanning planes, which were compared with a gold-standard of experienced radiographers who had access to the full conventional range of localiser images. 130 scans were used as a testing set.

Task 2: We trained a neural network on 1,500 transaxial stack slices to perform automated segmentation of important cardiac structures, great vessels, and other pathologies. The system assembled a 3D model of the thorax from which it made clinical measurements which were compared to those

Abstract 7 Table 1 Associations between selected patient characteristics and myocardial extracellular volume (ECV) at baseline

\begin{tabular}{|c|c|c|c|c|c|c|}
\hline \multirow[t]{2}{*}{ Baseline Covariate } & \multicolumn{3}{|c|}{ Univariable Model } & \multicolumn{3}{|c|}{ Multivariable Model } \\
\hline & $\beta$-coefficient & $95 \% \mathrm{Cl}$ & P-value & $\beta$-coefficient & $95 \% \mathrm{Cl}$ & P-value \\
\hline $\mathrm{BMI}-\mathrm{kg} / \mathrm{m}^{2}$ & $-0.18(0.05)$ & -0.28 to -0.08 & $<0.001$ & $-0.19(0.05)$ & -0.29 to -0.10 & $<0.001$ \\
\hline Haemoglobin - g/dL & $-0.49(0.20)$ & -0.89 to -0.09 & 0.02 & $-0.69(0.18)$ & -1.05 to -0.34 & $<0.001$ \\
\hline LA strain (reservoir) $-\%$ & $-0.13(0.04)$ & -0.20 to -0.06 & $<0.001$ & $-0.11(0.04)$ & -0.20 to -0.03 & 0.01 \\
\hline Aortic distensibility $-10^{-3} / \mathrm{mmHg}$ & $0.43(0.35)$ & -0.27 to 1.13 & 0.22 & $0.69(0.29)$ & 0.11 to 1.27 & 0.02 \\
\hline
\end{tabular}

$\mathrm{BMI}$ - body mass index; $\mathrm{Cl}$ - confidence interval; $\mathrm{LA}$ - left atrial. 